IOS Press

\title{
Effects of balance training on post-sprained ankle joint instability
}

\author{
I. Faizullin a,b,* and E. Faizullina ${ }^{\mathrm{b}}$ \\ ${ }^{a}$ University of Erlangen-Nuremberg, Institute of Sport Science and Sport, International Master "Physical \\ Activity and Health", Erlangen, Germany \\ ${ }^{\mathrm{b}}$ Kazan State Medical University, Department of Dermatology, Venereology and STD, Kazan, Russia \\ *Corresponding author. E-mail: iaroslav.faizullin@gmail.com
}

BACKGROUND: Ankle sprain is a medical condition when ankle ligaments are totally or partially torn. The primary cause of ankle sprain is sharp movements like turning or rolling the foot [1]. The ankle sprain needs to be treated right after the trauma, because if not treated it could lead to decreased stability of the ankle joint and lead to chronic ankle instability, which is characterized by increased risk of the ankle sprain [2]. We suppose that rehabilitation after the ankle sprain could significantly increase the performance of sportsmen.

OBJECTIVE: To investigate effects of balance exercise training on instable ankle due to the previous ankle sprain injury. In addition, the secondary aim of this systematic review was to find the effectiveness of different balance training exercises on instable ankle in order to find better opportunities for rehabilitation of sportsmen.

METHODS: The studies were selected from PubMed and Scopus using the library of the FriedrichAlexander University of Erlangen-Nuremberg (further- UB FAU), we used full texts, and only texts in English were included in this review. The literature search was conducted at the end of December 2014. Texts included randomised controlled trials, which were published in the last 5 years (2009-2014). The literature was included in this review only if it was published in English and if the randomised controlled trial was conducted in the study and if the full text was available from UB FAU. The articles, which were found only in PubMed search, were excluded during Scopus search.

PubMed search. First MeSH term was "Balance training for the ankle sprain" and 44 articles were found in PubMed. Then 29 articles were filtered by title and excluded from the study. Remaining 15 articles were assessed reading their abstracts, 6 of them were excluded and only 4 articles were left. The second MeSH term was "Balance training for ankle injury". Four additional articles were found by initial search. Two of them were filtered by the title and 2 were excluded at the stage of reading abstracts. The third MeSH term was "Balance exercises for instable ankle". One additional article was found by initial search and was excluded after reading the abstract.

Scopus search. The same MeSH terms were used as in PubMed search. With the first MeSH term one article was found and filtered by the title. With the second MeSH term no results were found in the initial search and with the third term 2 articles were picked up by the initial search. One of these articles was filtered by the title. The other one was filtered after reading the abstract. Overall 8 articles were taken into consideration for conducting a systematic review. Nevertheless, three of them could not be 
downloaded for free even using UB FAU up to the 28th of December, 2014. Thus, five articles were taken for the systematic review. After reading all 5 articles, one article by Maraike Alice Wortmann and Carrie L. Docherty was excluded from the study because it was a systematic review per se and at the same time it was not mentioned neither in the article title, nor in the abstract that the current study was a systematic review [3]. Also the article by Borreani et al. 2014 [4] was excluded after reading the paragraph "Methods" as this was not an RCT but a descriptive study. Therefore, 3 articles were taken for conducting a systematic review.

RESULTS: The first article by Janssen et al. 2011 [5] was a 3-way randomised controlled trial with 1 year follow-up. Participants aged from 12 to 70 years used this intervention. People with active participation in sports with a lateral ankle sprain during the last 2 months were eligible for inclusion in the study. Participants were divided into 3 groups. Group 1 undertook an 8 week neuromuscular training programme. Group 2 wore sports brace during their sport activities for the duration of 1 year, and group 3 was a control group and used the combination of neuromuscular training program and wore sports brace for 8 weeks. There were 122 participants in the neuromuscular training group, 126 in the brace group and 136 in combined group. The drawback of this intervention was that there was no control over the care provid.

In the second study by Ben Moussa Zouita, A et al. 2013 [6] the objective was to investigate how the proprioceptive exercises effect the postural balance and isokinetic strength in athletes with ankle sprain. 16 participants were recruited in the study and divided into two groups. The experimental group consisted of 8 participants with unilateral ankle sprain symptoms. The control group included another 8 participants with bilateral non-injured ankles. The training programme included 24 sessions during 8 weeks, every session lasted between 20 and 30 minutes. Four prescribed exercises were used during the intervention: one exercise without any material, one exercise with a ball only, one exercise with a balance board only and one exercise with a ball and a balance board. As a result, after 8 weeks of proprioceptive rehabilitation a significant improvement in extensor and flexor strength of ankle at a speed of $60-\mathrm{deg} / \mathrm{sec}$ was registered.

The third study by Emery, Meeuwisse 2010 [7] was aimed to examine the effectiveness of the neuromuscular prevention strategy in youth soccer players. The inclusion criteria were adolescents between 13 and 18 years of age. The exclusion criteria were injury within 6 weeks and the history of systemic disease in anamnesis (i.e. cerebral palsy, head injury). 82 soccer teams were invited to take part in the intervention. 12 trainers declined the invitation, other 10 teams declined participation. Overall 60 teams took part in the intervention programme. The teams were randomised by a club. 32 training group teams ( $n=380$ players) and 28 control group players ( $n=364$ players) took part in the intervention. The training programme included dynamic stretching exercises, agility, jumping and balance and eccentric strength. The control programme was a standardized warm-up including static, dynamic and aerobic components and home-based stretching programme using 16-inch diameter wobble board used for 15 minutes during exercises. The injury rate in the training group was 2.08 injuries/1000 player-hours, and in the control group 3.35 injuries/1000 player-hours. The neuromuscular training programme was protective in injuries of youth soccer players.

CONCLUSIONS: Balance training is an effective training method for rehabilitation of instable ankle. Different approaches to balance training provide in general similar improvement for sprained ankle.

Implications for future studies: More RCTs on chronic ankle instability are needed with large sample size and use of different intensities of exercises. It would be better for the UB FAU to provide access to articles so that students and researches could download articles for free from different electronic sources. 
Keywords: Ankle sprain, balance training, ankle injury, instable ankle

Conflicts of interest statement: The authors declare that there are no conflicts of interest.

\section{References}

[1] American Academy of Orthopaedic Surgeons (2014): Sprained Ankle. Your connection to expert orthopaedic information. Available from: http://orthoinfo.aaos.org/topic.cfm?topic $=$ A00150

[2] Coughlin, Michael J.; Saltzman, Charles L.; Anderson, Robert B.; Mann, Roger A. (op. 2014): Mann's surgery of the foot and ankle. 9th ed. Philadelphia: Saunders/Elsevier.

[3] Wortmann, Maraike Alice; Docherty, Carrie L. (2013): Effect of balance training on postural stability in subjects with chronic ankle instability. In J Sport Rehabil 22 (2), pp. 143-149

[4] Borreani, Sebastien; Calatayud, Joaquin; Martin, Julio; Colado, Juan Carlos; Tella, Victor; Behm, David (2014): Exercise intensity progression for exercises performed on unstable and stable platforms based on ankle muscle activation. In Gait \& posture 39 (1), pp. 404-409. DOI: 10.1016/j.gaitpost.2013.08.006

[5] Janssen, Kasper W.; van Mechelen, Willem; Verhagen, Evert Alm (2011): Ankles back in randomized controlled trial $(\mathrm{ABrCt})$ : braces versus neuromuscular exercises for the secondary prevention of ankle sprains. Design of a randomised controlled trial. In BMC musculoskeletal disorders 12, p. 210. DOI: 10.1186/14712474-12-210

[6] Ben Moussa Zouita, A; Majdoub, O.; Ferchichi, H.; Grandy, K.; Dziri, C.; Ben Salah, F Z (2013): The effect of 8-weeks proprioceptive exercise program in postural sway and isokinetic strength of ankle sprains of Tunisian athletes. In Annals of Physical and Rehabilitation Medicine 56 (9-10), pp. 634-643. DOI: 10.1016/j. rehab.2013.08.003

[7] Emery, C.A.; Meeuwisse, W.H. (2010): The effectiveness of a neuromuscular prevention strategy to reduce injuries in youth soccer: A cluster-randomised controlled trial. In British journal of sports medicine 44 (8), pp. 555-562. DOI: $10.1136 / \mathrm{bjsm} .2010 .074377$ 